\title{
Morphometry of pulmonary arteries from angiograms in chronic obstructive lung disease
}

\author{
KEITH HORSFIELD AND MICHAEL THOMAS \\ From The Midhurst Medical Research Institute, Midhurst, West Sussex
}

ABSTRACT The application of morphometric techniques based on Strahler orders to the study of $\omega^{\circ}$ pulmonary angiograms is described. When the pulmonary arterial tree is ordered by Strahler's method, peripheral branches have the lowest orders and the main pulmonary artery the highest order. The 9 mean diameter of vessels in each order can then be determined. Pulmonary angiograms were obtained $\vec{r}$ from 16 patients, 10 of whom had chronic obstructive lung disease (COLD), the other six having normal angiograms. Six orders of branching were found in vessels of $1 \mathrm{~mm}$ diameter or greater, and $\mathrm{a}_{-}$ plot of log mean diameter versus order from the normal angiograms was linear. The mean diameters $\biguplus_{\infty}$ of orders 2,3 , and 4 (diameter 2 to $7 \mathrm{~mm})$ from COLD patients were significantly reduced $\left((\mathrm{p}<0 \cdot 01)^{.}\right.$ and the log mean diameter versus order plot was concave upwards. These changes were more marked when TLC was raised than when it was normal. Plots of diameters of vessels from zones of the lung in which the pathology was well advanced (as judged by radiological changes) showed even greatero reduction in the middle orders. These changes are probably the result of stretching of vessels in $\frac{\mathbb{D}}{\mathbb{D}}$ emphysematous lesions and diminution of blood flow from loss of capillary bed.

In pulmonary emphysema there is destruction of alveolar walls with loss of the accompanying capillary bed. Changes in the small arteries also occur and these have been investigated by microangiographic techniques. Braachi et al ${ }^{1}$ reviewed this topic and described a reduction in the number and size of intralobular arterial branches, loss of capillary haze, displacement and distortion of vessels, and the presence of $\mathrm{A}-\mathrm{V}$ anastomoses. Loss of peripheral vessels may be seen on the chest radiographs of some patients with chronic obstructive lung disease (COLD), and reduction in the diameter of mediumsized vessels has also been described but not well quantified. ${ }^{2} 3 \mathrm{~A}$ method of quantifying the morphology of the pulmonary arteries using polyester resin casts and injection techniques was described by Singhal et $a l^{4}$ and Horsfield. ${ }^{5}$ The purpose of this investigation was to apply the method to the study of pulmonary angiograms from patients with and without COLD, so as to quantify changes in the diameters of pulmonary arteries from the hilum down to $1 \mathrm{~mm}$ diameter vessels.

Address for reprint requests: Dr K Horsfield, The Midhurst Medical Research Institute, Midhurst, West Sussex GU29 0BL.

\section{Methods}

Pulmonary angiograms were obtained in 16 patients as part of the clinical investigation of dyspnoea, $\times$ chest pain, or suspected pulmonary embolism. The 3 purpose of the investigation and its nature were explained to the patients who gave their consent? to it. Ten transpired to have COLD and the remaining six had normal pulmonary angiograms, judged on the basis of complete vascular filling, transit time of the contrast medium, gradual decrease in vessel diameter towards the periphery, and the vascular N pattern. Lung function tests were performed in the COLD patients, but not routinely in the other six without COLD. Basic data for each patient are given $\omega$ in table 1. Cardiac catheterisation was performed with the patient sedated with $10 \mathrm{mg}$ diazepam and lying supine. The catheter was introduced via an antecubital vein and pulmonary artery pressureswere recorded in all but one (who did not have ${ }^{-}$ COLD), taking zero $5 \mathrm{~cm}$ below the sternal angle. $\frac{\text { Pे }}{\mathrm{D}}$ Seventy $\mathrm{ml}$ of contrast medium (Urografin) was? injected into the pulmonary artery at $34 \mathrm{ml} / \mathrm{s}$ using $\mathscr{\mathbb { D }}$ a Cordis automatic injector, and plates were exposed in an anterior-posterior projection up to a rate of $5 / \mathrm{s}$. 
Table 1 Age, sex, and diagnosis of patients studied

\begin{tabular}{llll}
\hline Patient & Sex & Age $(y r)$ & Diagnosis \\
\hline CS & M & 50 & Dyspnoea (psychogenic) \\
KM & F & 42 & ? pulmonary embolism \\
JB & F & 23 & ? pulmonary embolism \\
HB & M & 56 & Chest pain (cause unknown) \\
NA & M & 44 & Cough (cause unknown) \\
LR & F & 67 & Lymphoma \\
AJ & M & 65 & COLD \\
BB & M & 68 & COLD \\
GF & M & 72 & COLD \\
HW & M & 48 & COLD \\
AD & M & 48 & COLD \\
JM & M & 70 & COLD \\
JJ & F & 63 & COLD \\
AH & M & 57 & COLD \\
RM & F & 75 & COLD \\
DC & M & 56 & COLD \\
\hline
\end{tabular}

The distance from the focal spot to the plate was 100 $\mathrm{cm}$, and the patient's back was $2 \mathrm{~cm}$ above the plate.

The best plate from the series was chosen by inspection, taking into account completeness of arterial filling and contrast. A copy of the plate was made and returned to the file, while the original was kept for study. It was laminated in thin, transparent plastic sheeting and sealed, so that ink markings could be made on the surface and erased if necessary without damaging the film. The plate was studied on a horizontal viewer. Arterial branches were ordered by the method of Strahler, ${ }^{6} 7$ originally described for the study of rivers. The most peripheral (smallest) branches are order 1, and two of these meet to form an order 2 branch. An order 3 branch is formed when two order 2 branches meet, and so on up to the main stem or river outlet. When branches of differing order meet, the parent branch continues with the same order as the higher ordered daughter branch. Finally, contiguous branches of the same order are considered to constitute just one branch of that order (fig 1). The application of the method to the study of the pulmonary arteries is described by Singhal $e t a l^{4}$ and Horsfield. ${ }^{5}$

An order 1 branch was defined arbitrarily as the first branch on any pathway, from the periphery towards the main pulmonary trunk, the diameter of which was equal to or greater than $1 \mathrm{~mm}$. Measurements were made by placing a transparent ruler directly on the plate; it was calibrated in millimetre divisions and read to the nearest $0.2 \mathrm{~mm}$ as judged by eye. When the order 1 branches had been identified, the outline of the arterial tree was traced with a fine drawing pen, the order of each branch determined, and the order identified with a colour code. The main pulmonary trunk was excluded from the study because its borders were not sufficiently well visualised on some of the angiograms for the diameter to be measured accurately. The diameter of each branch was measured in the middle of its length,

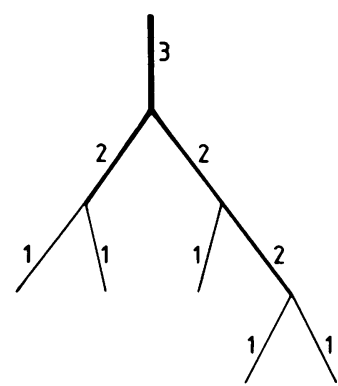

(a)

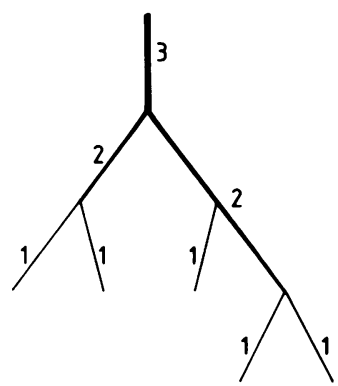

(b)
Fig 1 Strahler orders. (a) First stage of attribution of orders. (b) Second stage, in which contiguous branches of the same order become just one branch (note order 2 branches on right of figure).

taking that of the upstream segment when contiguous segments of equal order occurred. Finally, the mean diameter of branches in each order was calculated. Although this was done for the whole tree, it was instructive to divide the lung into upper and lower zones and to calculate the mean values for each zone separately. When mean diameter is plotted on a logarithmic scale against order, a linear relation is usually obtained (this applies to most structures, not only to arteries). Thus mean diameter increases geometrically with order. Lengths were not measured because unless a branch runs in a plane at right angles to the projection it is foreshortened. Diameter measurements are not subject to this error providing segments of vessel are cylindrical.

Differences between diameters in each group were subject to the Wilcoxon two-sample rank test. ${ }^{8}$ This is a non-parametric test in which the values for the two groups are combined and ranked in order of magnitude. The rank order is determined for each value and the sum of the ranks for the smaller group obtained. $T^{1}$ is calculated from

$$
\mathrm{T}^{1}=\mathrm{N} 1(\mathrm{~N} 1+\mathrm{N} 2+1)-\mathrm{T}
$$

where $\mathrm{N} 1$ is the number in the smaller group and $\mathrm{T}$ is the total of rank numbers in that group, and N2 is the number in the larger group. A table is entered with the smaller of $T$ and $T^{1}$, which gives the value at which the null hypothesis is rejected. A particular value of this method is that no assumptions need to be made regarding the form of the distribution.

\section{Results}

Table 2 gives the systolic, diastolic, and mean pulmonary artery pressures. In one patient (JB) a technically inadequate record was obtained, and in another (LR) the pressures were not measured. Pressures were thus obtained in four of the patients with normal angiograms, the mean systolic and 
Table 2 Height, weight, pulmonary function tests, and pulmonary artery pressures in COLD patients

\begin{tabular}{|c|c|c|c|c|c|c|c|c|c|c|c|}
\hline Patient & $\begin{array}{l}\text { Height } \\
(m)\end{array}$ & $\begin{array}{l}\text { Weight } \\
(k g)\end{array}$ & $\begin{array}{l}T L C \\
(l)\end{array}$ & $\begin{array}{l}V C \\
(l)\end{array}$ & $\begin{array}{l}R V \\
(l)\end{array}$ & $\begin{array}{l}R V \mid T L C \\
(\%)\end{array}$ & $\begin{array}{l}F E V_{1} \\
(l)\end{array}$ & $\begin{array}{l}F E V_{1} \\
(\%)\end{array}$ & $\begin{array}{l}\text { Raw } \\
\left(\mathrm{cmH}_{2} \mathrm{O} / \mathrm{l} / \mathrm{s}\right)\end{array}$ & $\begin{array}{l}V \text { effic } \\
(\%)\end{array}$ & $\begin{array}{l}P A P \\
(m m H g)\end{array}$ \\
\hline $\mathbf{A J}$ & $1 \cdot 59$ & 76 & $5 \cdot 30$ & 1.96 & $3 \cdot 34$ & 63 & 0.92 & 43 & $2 \cdot 29$ & 32 & $28 / 11(17)$ \\
\hline BB & $1 \cdot 71$ & 65 & $7 \cdot 93$ & $2 \cdot 11$ & $5 \cdot 82$ & 73 & 0.51 & 38 & $4 \cdot 24$ & 36 & $30 / 18$ (22) \\
\hline GF & 1.62 & 64 & $5 \cdot 05$ & 2.09 & 2.96 & 59 & 0.57 & 30 & $2 \cdot 75$ & 36 & $23 / 12$ (16) \\
\hline HW & $1 \cdot 62$ & 53 & $8 \cdot 88$ & $1 \cdot 84$ & $7 \cdot 04$ & 79 & 0.64 & 41 & $4 \cdot 21$ & 26 & $24 / 12$ (16) \\
\hline AD & 1.66 & 60 & $6.33 \dagger$ & $*$ & * & $*$ & 0.84 & 45 & $4 \cdot 20$ & 27 & $32 / 12(20)$ \\
\hline $\mathbf{J M}$ & 1.66 & 62 & $8 \cdot 71$ & $2 \cdot 80$ & 5.97 & 68 & 0.93 & 65 & $6 \cdot 43$ & 31 & $40 / 20$ (26) \\
\hline JJ & 1.66 & 43 & $6 \cdot 21$ & $1 \cdot 62$ & $4 \cdot 59$ & 74 & 0.55 & 42 & 4.67 & 22 & $35 / 15(25)$ \\
\hline AH & 1.67 & 51 & $7 \cdot 47$ & $2 \cdot 74$ & $4 \cdot 73$ & 63 & 0.62 & 25 & $5 \cdot 74$ & 35 & $36 / 12(20)$ \\
\hline RM & 1.65 & 56 & $6 \cdot 79$ & 1.83 & 4.96 & 73 & 0.97 & 58 & $3 \cdot 17$ & 48 & $30 / 10(18)$ \\
\hline DC & 1.67 & 45 & $6 \cdot 76$ & $2 \cdot 28$ & $4 \cdot 48$ & 66 & 0.69 & 30 & 3.08 & 33 & $32 / 15$ (25) \\
\hline
\end{tabular}

* Not measured; †measured by nitrogen washout volume; $T L C=$ total lung capacity using thoracic gas volume; VC $=$ vital capacity; $R$ V = residual volume; $R V / T L C=$ ratio expressed as per cent; $F E V_{1}=$ forced expiratory volume in one second; $F E V / F V C=$ ratio of $F E V$, to forced vital capacity as per cent; Raw =airways resistance; $\mathrm{V}$ effic $=$ ventilatory efficiency; $\mathbf{P A P}=$ pulmonary artery pressure, systolic $/$ diastolic (mean); $1 \mathrm{cmH}_{2} \mathrm{O} / 1 / \mathrm{s}=0.098 \mathrm{kPal}^{-1} \mathrm{~s} ; 1 \mathrm{mmHg}=0.133 \mathrm{kPa}$.

diastolic values for the group being $23.0 / 6.0 \mathrm{mmHg}$ $(3.06 / 0.80 \mathrm{kPa})$ and the mean pressure $12.25 \mathrm{mmHg}$ $(1.63 \mathrm{kPa})$. In the subjects with COLD the mean systolic and diastolic pressures for the group were about $8 \mathrm{mmHg}(1.07 \mathrm{kPa})$ higher at $31.0 / 13.7 \mathrm{mmHg}$ $(4 \cdot 13 / 1.83 \mathrm{kPa})$ while the mean pressure was 20.5 $\mathrm{mmHg}(2.73 \mathrm{kPa})$. Table 2 also gives the pulmonary function test results for the COLD patients. With the exception of normal total lung capacity in patients AJ, BB, and GF, all showed the changes expected in COLD. Total lung capacity (TLC), residual volume, residual volume/TLC ratio, and airways resistance were raised, and vital capacity, forced expiratory volume in one second, and ventilatory efficiency ${ }^{9}$ were reduced. Although a clinical diagnosis of emphysema was made in these patients, the term is not used in this paper because emphysema is strictly a structural diagnosis, requiring anatomical investigation to confirm it. Table 3 gives the mean and range of the diameters for each order. This is divided into four groups: those with normal angiograms, those with COLD and normal TLC, those with COLD and raised TLC, and the two previous

Table 3 Mean and range of mean diameters ( $\mathrm{mm})$ for each order in the normal angiograms, and in the angiograms from the patients with COLD

\begin{tabular}{|c|c|c|c|c|}
\hline Order & $\begin{array}{l}\text { Angiograms } \\
\text { normal }\end{array}$ & $\begin{array}{l}\text { COLD with } \\
\text { TLC normal }\end{array}$ & $\begin{array}{l}C O L D \text { with } \\
T L C \text { raised }\end{array}$ & $\begin{array}{l}C O L D \\
\text { all cases }\end{array}$ \\
\hline 1 & $\begin{array}{l}1 \cdot 2 \\
(1 \cdot 1-1 \cdot 3)\end{array}$ & $\begin{array}{l}1 \cdot 3 \\
(1 \cdot 2-1 \cdot 4)\end{array}$ & $\left(\begin{array}{l}1 \cdot 2 \\
(1 \cdot 1-1 \cdot 2)\end{array}\right.$ & $\begin{array}{l}1 \cdot 2 \\
(1 \cdot 1-1 \cdot 4)\end{array}$ \\
\hline 2 & $\left(\begin{array}{l}2 \cdot 2 \\
(1 \cdot 9-2 \cdot 3)\end{array}\right.$ & $(1 \cdot 9-2 \cdot 3)$ & $(1 \cdot 8-2 \cdot 1)$ & $\begin{array}{l}1 \cdot 9 \\
(1 \cdot 6-2 \cdot 3)\end{array}$ \\
\hline 3 & $\left(\begin{array}{l}3 \cdot 8 \\
(3 \cdot 5-4 \cdot 2)\end{array}\right.$ & $\begin{array}{l}3 \cdot 6 \\
(3 \cdot 3-3 \cdot 8)\end{array}$ & $\begin{array}{l}3 \cdot 0 \\
(2 \cdot 7-3 \cdot 8)\end{array}$ & $\begin{array}{l}3 \cdot 2 \\
(2 \cdot 7-3 \cdot 8)\end{array}$ \\
\hline 4 & $\begin{array}{l}7 \cdot 0 \\
(5 \cdot 7-8 \cdot 4)\end{array}$ & $\begin{array}{l}6 \cdot 3 \\
(4 \cdot 9-7 \cdot 2)\end{array}$ & $\begin{array}{l}5 \cdot 2 \\
(4 \cdot 5-5 \cdot 9)\end{array}$ & $\begin{array}{l}5 \cdot 5 \\
(4 \cdot 5-7 \cdot 2)\end{array}$ \\
\hline 5 & $\begin{array}{l}14 \cdot 7 \\
(12 \cdot 0-18 \cdot 4)\end{array}$ & $\begin{array}{l}17 \cdot 4 \\
(12 \cdot 7-23 \cdot 8)\end{array}$ & $\begin{array}{l}13 \cdot 6 \\
(8 \cdot 0-21 \cdot 0)\end{array}$ & $\begin{array}{l}14 \cdot 7 \\
(8 \cdot 0-23 \cdot 8)\end{array}$ \\
\hline 6 & $\begin{array}{l}27 \cdot 5 \\
(25 \cdot 1-32 \cdot 5)\end{array}$ & $\begin{array}{l}32 \cdot 2 \\
(27 \cdot 5-35 \cdot 0)\end{array}$ & $\begin{array}{l}29 \cdot 2 \\
(25 \cdot 0-32 \cdot 6)\end{array}$ & $\begin{array}{l}30 \cdot 1 \\
(25 \cdot 5-35 \cdot 0)\end{array}$ \\
\hline
\end{tabular}

TLC $=$ total lung capacity. groups combined, namely all those with COLD. Table 4 gives the statistical significance of the differences between the groups for each order. It can be seen that the most significant differences are between the normal group and those with COLD and a raised TLC, orders 2,3 , and 4 being significantly smaller in the latter group $(p<0.01)$. These smaller diameters in the middle range of orders should result in the log mean diameter against order plots in COLD becoming concave upwards. In order to make this change in shape easier to see, and to facilitate comparison with the normal group, the data were normalised. This was done for each individual separately by taking the logarithms of the diameters, subtracting the logarithm of the diameter of the order 1 branch from each, and then multiplying by $\log 30 /(\log \mathrm{D}$ order $6-\log \mathrm{D}$ order 1$)$. Finally the antilogs of the numbers thus obtained were recorded. By this method order 1 is normalised to $1.0 \mathrm{~mm}$ diameter, order 6 to $30 \mathrm{~mm}$ diameter, and the others are adjusted proportionally, maintaining the shape of the plot. Figure 2 shows the plots of $\log$ mean normalised diameters against order. The normal angiograms give an almost linear plot,

Table 4 Significance levels (value of $p$ ) for the differences in diameters of each order, for the same four groups shown in table 3 using Wilcoxon's two-sample rank test

\begin{tabular}{lllllll}
\hline Category & \multicolumn{9}{l}{ Order } \\
\cline { 2 - 7 } & $I$ & 2 & 3 & 4 & 5 & 6 \\
\hline Normal-all COLD & NS & NS & 0.01 & 0.05 & NS & NS \\
Normal_COLD, TLC N & NS & NS & NS & NS & NS & NS \\
Normal_COLD, TLC R & NS & 0.01 & 0.01 & 0.01 & NS & NS \\
COLD, TLC N-COLD, & NS & NS & 0.05 & NS & NS & NS \\
TLC R & & & & & & \\
\hline
\end{tabular}

$\mathrm{NS}=$ not significant, $\mathrm{p}>0.05$.

TLC N = total lung capacity within $95 \%$ confidence limits of normal range.

TLC $\mathbf{R}=$ total lung capacity raised. 


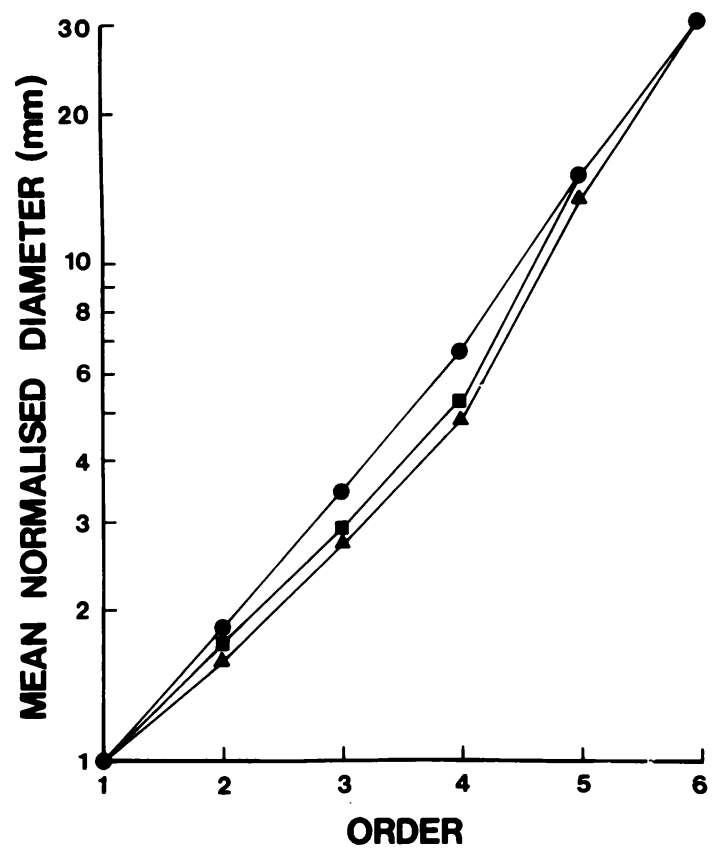

Fig 2 Mean normalised data from normal angiograms and angiograms from $C O L D$ patients. Ordinate, mean normalised diameter on a logarithmic scale. Abscissa, Strahler order. Onormal, $\square C O L D$ with normal $T L C, \triangle C O L D$ with raised $T L C$.

whereas the angiograms from COLD patients are concave upwards. COLD patients with a raised TLC have a greater reduction in the diameter of the middle orders than do COLD patients with a normal TLC. When interpreting fig 2 the logarithmic scale on the ordinate should be borne in mind, because this makes the differences between the plots appear to be less than they actually are.

\section{Discussion}

Compared with arterial casts, angiograms have the advantage of representing vessels in situ during life. They do, however, have some disadvantages. With an anterior-posterior projection the magnification of objects situated in the middle of the chest is $18 \%$, and those vessels situated anteriorly are more magnified than those situated posteriorly. This difference amounts to $7 \%$. Corrections for the $18 \%$ magnification were not made, as in this paper we are describing the angiographic appearances, but readers wishing to do this can multiply the stated diameters by $100 / 118$.

A check was made on the accuracy of the diameter measurements. An excised human lung was inflated with saline from a pump at a constant pressure of $20 \mathrm{cmH}_{2} \mathrm{O}$, and the pulmonary artery was injected with a mixture of gelatine and barium sulphate. Formalin $40 \%$ was then added to the perfusate to make a $2 \%$ formalin solution, which fixed the lungs in the inflated position and solidified the gelatin. A radiograph of the lung was taken and the diameters of a selection measured on the plate. Finally, the relevant parts of the pulmonary arterial tree were carefully dissected and the diameters of the vessels measured directly. Results were within 0.5 to $1.0 \mathrm{~mm}$ for vessels of $5-10 \mathrm{~mm}$ diameter-that is, within $10 \%$. This represents fairly good agreement when allowance is made for magnification on the plate of $5 \%$ for structures at the hilum, and for wall thickness of about $2 \times 8 \%$ to be subtracted from the external diameter of the dissected vessels.

Given a range of error of diameter of $\pm 7 \%$, what effect would this have on the results? In discussing this problem the overall magnification of $18 \%$ will be ignored and vessels in the plane of the hilum will be assumed to be measured accurately. Vessels of $1.0 \mathrm{~mm}$ diameter (those from which the ordering process starts) would appear to be from 0.93 to $1.07 \mathrm{~mm}$, and would be recorded as $1.0 \mathrm{~mm}$ so that order 1 vessels would not be significantly affected. Since all the other orders follow automatically once the order 1 branches have been defined, the overall ordering should not be in error because of this. Larger branches of say $6.0 \mathrm{~mm}$ diameter would appear to be from 5.6 to $6.4 \mathrm{~mm}$ and be recorded as 5.5 to $6.5 \mathrm{~mm}$; thus a few of these might be up to $0.5 \mathrm{~mm}$ in error. Larger branches lie much more nearly to the plane of the hilum and are not therefore subject to such variable degrees of magnification. Thus the medium-sized vessels have the largest range of error, but some of these will be positive and some negative, so that the difference in the mean values is unlikely to be seriously affected. Furthermore, taking as an example the mean diameter of an order 4 vessel as $5.55 \mathrm{~mm}$, an error of $\pm 10 \%$ lies well within the range of diameters for this order, and well outside the ranges for order 3 and order 5 vessels.

For the most part determination of Strahler order presented no difficulty, but some difficulties were found with many of the angiograms. These related to the superimposition of vascular images in such a way that it was difficult to determine their precise pattern of branching. Most of the doubts arose with vessels fairly close to the hilum, especially of the left lung, and comparison with the branching pattern of arterial casts often proved helpful. If it was still difficult to come to a decision, other angiograms from the series, both earlier and later, were studied. Finally, if it was impossible to be sure of the branching pattern, arteries peripheral to the branch at issue 
were excluded from the analysis. Fortunately, the total number of orders in the tree was not sensitive to these uncertainties, which involved decisions such as whether a branch joining an order 6 vessel was itself of order 4 or order 5 . In neither case would this change the order 6 branch. Although these difficulties in determining order resulted in the occasional loss of some data, there is no reason to believe that there was any trend towards loss of either high or low diameter vessels, and it seems unlikely that the mean values were materially affected.

Another possible source of error in the measurement of angiograms from patients with COLD is failure of vessels to fill. In this case, however, abnormal vessels would be most likely to be affected, resulting in an underestimate of the magnitude of the changes found.

The plot of log mean diameter against order (fig 2) for the normal angiograms is almost linear, in agreement with the findings from the cast study. ${ }^{4}$ In the cast there were seven orders of branching, from arteries of $1.3 \mathrm{~mm}$ diameter (mean) to the main pulmonary artery of $30 \mathrm{~mm}$ diameter. The angiograms showed six orders of branching from arteries of $1.2 \mathrm{~mm}$ diameter (mean) to the right or left main branch of the pulmonary artery of $27.5 \mathrm{~mm}$ diameter (mean). Considering the differences between the two techniques this is fairly good agreement, and allowing one order for the unmeasured pulmonary trunk in the angiogram data, both methods give seven orders. Thus the loss of some data because of difficulties in interpretation of the pattern of branching discussed above, did not affect the total numbers of orders in the system.

Within each subset of data (normal, COLD with normal TLC, COLD with raised TLC, and all COLD) there is an absolute separation of diameter values between orders-that is, the ranges of values for successive orders do not overlap (table 3 ). Comparing angiograms from COLD patients with raised TLC with the normal angiograms, order 2 arteries are reduced from 2.2 to $1.8 \mathrm{~mm}$, order 3 arteries from 3.8 to $3.0 \mathrm{~mm}$, and order 4 arteries from 7.0 to $5.2 \mathrm{~mm}$ (mean values). If resistance is inversely proportional to the radius (and hence the diameter) to the fourth power, then in order 2 arteries resistance is increased $\times 2$, in order 3 arteries $\times 2 \cdot 5$, and in order 4 arteries $\times 3$. Such changes would be expected to contribute to pulmonary hypertension only if they were widespread throughout the pulmonary arterial tree. In most cases, however, they were patchily distributed, some regions being severely affected and others being near normal, so that no great effect on pulmonary artery pressure would be expected. This is borne out by the values observed in the COLD patients.
The diminution of diameter in orders 2,3 , and $4 \vec{\Rightarrow}$ is greater when TLC is raised than when it is within the normal range, and this may represent stages in the disease. Narrowing of these vessels may be related to stretching in the presence of bullae and emphysematous spaces, so that the anatomical type $\stackrel{\mathbb{Q}}{\Omega}$ of emphysema may also be relevant. This point could \& only be demonstrated by necropsy studies of lung structure. Of the three patients with COLD and normal TLC, one (BB) was only just within the $95 \% \overrightarrow{\vec{\omega}}$ range of normal values and may well have had what was for him a raised value. Another (AJ) also had $\overrightarrow{\vec{x}}$ ankylosing spondylitis with fixed costovertebral $\dot{\omega}_{-}$ joints. This prevented his chest expanding so that or he was unable to develop a raised TLC in spite of $\omega$ severe lung disease. Thus two of the three had a 0 potentially raised TLC, which is probably reflected 은 in their log mean diameter against order plot (fig 2). This lies nearer to that for the other COLD patients than to that for the normal lungs.

Narrowing and loss of medium-sized pulmonary $\vec{\bullet}$ arteries has been described previously ${ }^{2} 3$ but not $\stackrel{\infty}{\rightarrow}$ well quantified. An increase in the diameter of hilar vessels may be found in COLD, and Simon ${ }^{3}$ suggested that this may be associated with pulmonary hypertension. In this study the normal order 6 vessels averaged $27.5 \mathrm{~mm}$ diameter, while those from patients with COLD averaged $30.1 \mathrm{~mm}$. The difference did not reach statistical significance. A mild degree of pulmonary hypertension was found in the patients with COLD, the systolic, diastolic, and mean pressures averaging $8 \mathrm{mmHg}(1.07 \mathrm{kPa})$ higher than in those with normal angiograms.

The results so far presented represent the mean values for the whole lung. Much more dramatic ? changes may be found when the pathological process predominantly affects one zone, and the results from this are compared with those from the more normal zone. Figure 3 shows the angiogram

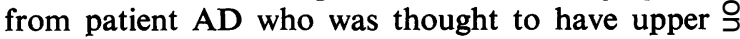
zone emphysema. Figure 4 gives the data plots $\rightarrow$ from this angiogram for the upper and lower zones separately, and although both plots are concave $\tilde{N}$ upwards, that for the upper zone is obviously more so. As with fig 2, the logarithmic scale on the ordinate should be borne in mind.

It seems likely that these changes in diameter of the medium-sized vessels accompany emphysema, $\stackrel{\bullet}{\circ}$ but a definitive statement on this point cannot be $\mathbb{\varnothing}$ made without necropsy studies of structure. How $\stackrel{\mathcal{P}}{\rightarrow}$ early these vascular changes occur in the disease $\frac{0}{0}$ process and their diagnostic importance remain to be determined.

We wish to thank S Phillips and W Kemp for their technical assistance. 


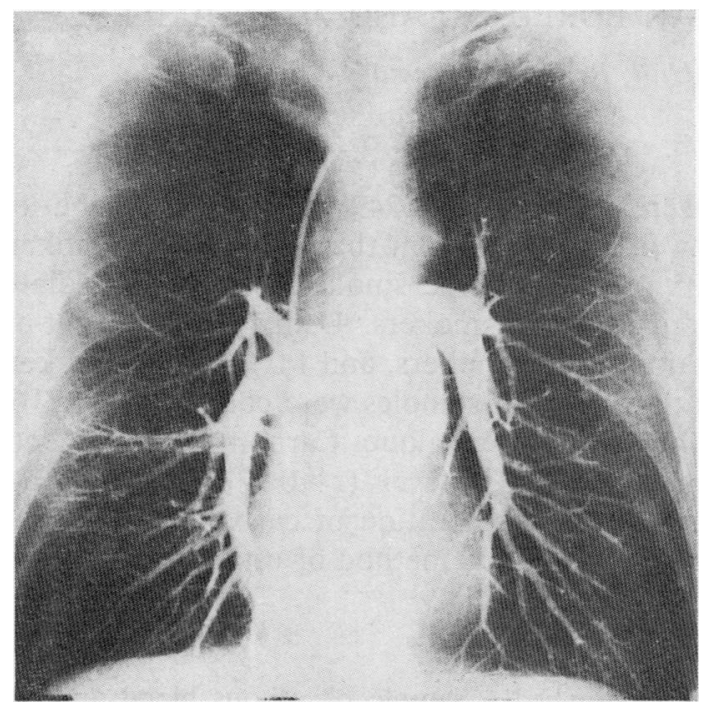

Fig 3 Angiogram from patient AD showing marked diminution of the diameters of vessels in the upper zones caused by emphysema.

\section{References}

1 Braachi G, Barbaccia P, Vezzoli F, Marazzini L, Longhini E. Peripheral pulmonary wedge angiography in chronic obstructive pulmonary disease. Chest 1977; 71:718-24.

2 Scarrow GD. The pulmonary angiogram in chronic bronchitis and emphysema. Clin Radiol 1966;17:54-67

3 Simon G. Principles of chest $x$-ray diagnosis. Fourth edition. London: Butterworths, 1978.

4 Singhal S, Henderson R, Horsfield K, Harding K, Cumming G. Morphometry of the human pulmonary arterial tree. Circ Res 1973; 33:190-7.

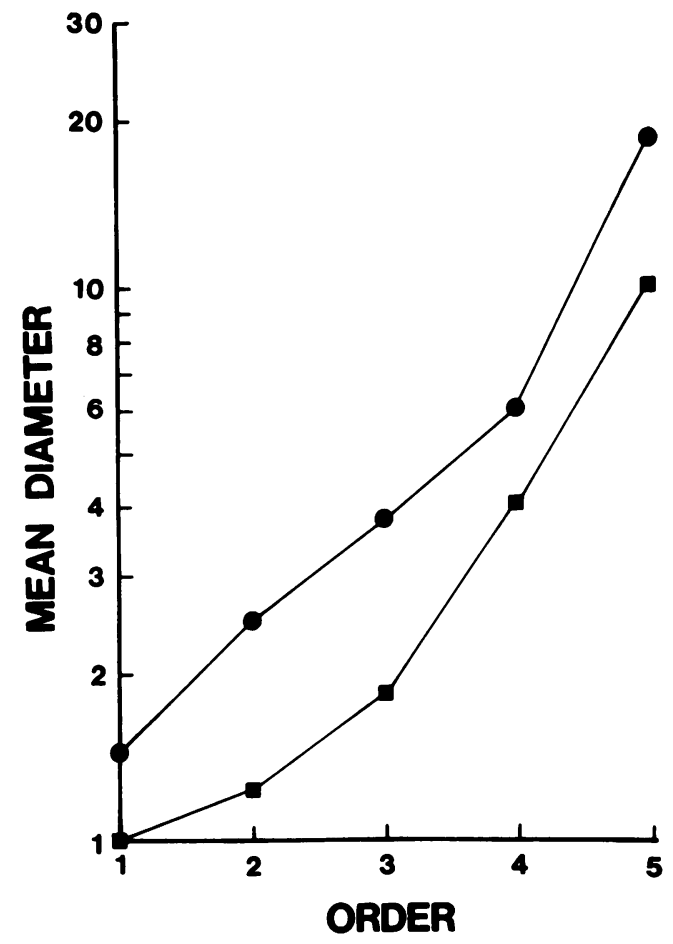

Fig 4 Mean data from the angiogram of patient $A D$, shown in fig 3. Ordinate, mean diameter on a logarithmic scale. Abscissa, Strahler order olower zone,

uupper zone.

5 Horsfield K. Morphometry of the small pulmonary arteries in man. Circ Res 1978; 42:593-7.

6 Strahler AN. Revisions of Horton's quantitative factors in erosional terrain (abstr). Trans Am Geophys Union 1953; 34 :345.

7 Strahler AN. Quantitative analysis of watershed geomorphology. Trans Am Geophys Union 1957; 38:913-20.

8 Snedecor GW, Cochran WG. Statistical methods. Sixth edition. Iowa: Iowa State University Press, 1967.

9 Cumming G. Gas mixing efficiency in the human lung. Respir Physiol 1967; 2:213-24. 